Cahiers
de a Recherche
sur les Droits Fondamentaux
Cahiers de la recherche sur les droits fondamentaux

$18 \mid 2020$

La vulnérabilité

Pour une justice pénale internationale en matière environnementale : à propos de la répression des atteintes à l'environnement par une juridiction internationale spécialisée

\title{
Mamoud Zani
}

\section{OpenEdition}

\section{Journals}

Édition électronique

URL : https://journals.openedition.org/crdf/6472

DOI : $10.4000 /$ crdf. 6472

ISSN : 2264-1246

Éditeur

Presses universitaires de Caen

Édition imprimée

Date de publication : 19 novembre 2020

Pagination : 123-132

ISBN : 978-2-84133-987-7

ISSN : 1634-8842

Référence électronique

Mamoud Zani, « Pour une justice pénale internationale en matière environnementale : à propos de la répression des atteintes à l'environnement par une juridiction internationale spécialisée », Cahiers de la recherche sur les droits fondamentaux [En ligne], 18 | 2020, mis en ligne le 19 novembre 2021, consulté le 14 novembre 2022. URL : http://journals.openedition.org/crdf/6472 ; DOI : https://doi.org/10.4000/ crdf.6472 


\title{
Pour une justice pénale internationale en matière environnementale: à propos de la répression des atteintes à l'environnement par une juridiction internationale spécialisée
}

\author{
Mamoud ZANI \\ Professeur de droit public \\ Directeur du Centre de droit international et européen (CDIE) de Tunis \\ Vice-président chargé de l'enseignement supérieur et de la recherche scientifique au Centre européen de recherche et de prospective politique (CEREPPOL)
}

I. La répression confinée des atteintes à l'environnement par la CPI
A. Les lacunes statutaires
B. Les améliorations nécessaires

II. La répression étendue des atteintes à l'environnement par des juridictions régionales

A. La Cour européenne de Strasbourg

B. La Cour africaine de Malabo

III. La répression effective des atteintes à l'environnement par de nouvelles initiatives normatives et juridictionnelles

A. La Convention internationale pour la lutte contre l'écocide

B. La Cour pénale internationale de l'environnement

La question de la criminalisation des atteintes portées à l'environnement par une juridiction internationale ne date point d'aujourd'hui. En effet, l'Organisation des Nations unies (ONU), à travers son organe de coordination ${ }^{1}$, le Conseil économique et social (ECOSOC), porta un intérêt particulier au rôle de la justice pénale dans la protection de l'environnement, notamment eu égard aux conséquences néfastes des catastrophes écologiques. Sur recommandation de la Commission pour la prévention du crime et la justice pénale ${ }^{2}$ l'ECOSOC adopta la résolution 1992/22 par laquelle il estima que «la criminalité nationale et transnationale, le crime organisé, les délits économiques, en particulier le blanchiment de l'argent et le rôle du droit pénal dans la protection de l'environnement $»^{3}$ devaient constituer des priorités absolues pour les travaux de ladite commission. Par la suite, l'ECOSOC, par le truchement de toute une série de résolutions ${ }^{4}$, ne fit que conforter son action en matière de protection de l'environnement.

1. Voir Charte des Nations unies, art. 63, al. 2.

2. Elle a été créée par l'ECOSOC comme commission technique dans sa résolution 1992/1. En 2006, l'Assemblée générale élargit son mandat en vertu de sa résolution $61 / 252$.

3. Voir doc. A/CONF/169/12, 14 décembre 1994, p. 3, \$2.

4. Résolutions $1993 / 32,1993 / 28$ et $1994 / 15$. 
Il en est ainsi de l'approbation du programme de travail du neuvième congrès de l'ONU pour la prévention du crime et le traitement des délinquants autour du thème «la protection de l'environnement aux échelons national et international: potentiel et limites de la justice pénale »; l'intégration des délits écologiques parmi les questions faisant l'objet de coopération technique; la prise en compte par les organes compétents de l'ONU et ses États membres des recommandations afférentes au rôle du droit pénal dans la sauvegarde de l'environnement, formulées par le Groupe spécial d'experts chargé d'examiner des formes plus efficaces de coopération internationale contre la criminalité transnationale, y compris les crimes contre l'environnement.

L'initiative d'une juridiction pénale internationale en matière environnementale fut relancée devant la Commission pour la prévention du crime et la justice pénale par le Costa Rica. Celui-ci proposa, en 1996, la création d'une Cour internationale sur l'environnement pour connaître des crimes écologiques internationaux, poursuivre les criminels et aider les États à interpréter les accords internationaux sur l'environnement ${ }^{5}$. Toutefois, la proposition en question est restée lettre morte faute de consensus entre États. Elle fut débattue aussi dans le cadre du projet de Code des crimes contre la paix et la sécurité de l'humanité, précurseur du Statut de la Cour pénale internationale (CPI). À cet effet, en procédant à l'analyse dudit projet, la Commission du droit international $(\mathrm{CDI})^{6}$ considéra la protection de l'environnement comme étant un intérêt fondamental de l'humanité et que, par conséquent, les atteintes particulièrement graves à cet intérêt devraient en bonne logique engager la responsabilité pénale internationale de leurs auteurs.

La première version du projet de Code de $1991^{7}$ prévoyait deux dispositions ayant trait à l'environnement: d'un côté, l'article 22, alinéa 2-d (crime de guerre d'une exceptionnelle gravité) concernant l'utilisation de méthodes ou moyens de guerre qui sont conçus pour causer, ou dont on peut attendre qu'ils causeront, des dommages étendus, durables et graves à l'environnement naturel; de l'autre, l'article 26 (dommages délibérés et graves à l'environnement) se rapportant à la condamnation de tout individu causant délibérément ou ordonnant que soient causés des dommages étendus, durables et graves à l'environnement naturel. Cet élan ambitieux fut par la suite freiné; les deux articles ont en effet été in globo altérés: la nouvelle mouture de l'article 22 proposée par le rapporteur spécial a supprimé toute référence à la notion d'environnement, outre que les membres de la CDI avaient insisté sur la suppression du membre de phrase «exceptionnelle gravité» qui suscite de vives inquiétudes s'agissant de sa signification et de son implication en droit international humanitaire ${ }^{8}$. En sus, la suppression de l'article 26 a révélé une dissension ${ }^{9} \mathrm{au}$ sein même de la CDI: certains membres affirmaient que les dommages à l'environnement ne devaient pas figurer dans le Code car ils ne répondaient pas aux critères de qualification des crimes contre la paix et la sécurité de l'humanité; par contre, d'autres membres avaient plaidé en faveur du maintien de l'article car les dommages délibérés et graves à l'environnement étaient en réalité non seulement pour l'humanité d'aujourd'hui mais également pour les générations futures.

Dans le même ordre d'idées, la première partie du «Projet d'articles sur la responsabilité des États» adopté à titre provisoire ${ }^{10}$, en première lecture, par la CDI, comprenait le problème des atteintes à l'environnement. À cet effet, l'article 19, alinéa 3 , d considérait comme crime international:

[...] une violation grave d'une obligation internationale d'importance essentielle pour la sauvegarde et la préservation de l'environnement humain, comme celles interdisant la pollution massive de l'atmosphère ou des mers ${ }^{11}$.

Mais ce maigre effort n'a pu se concrétiser ultérieurement puisque le projet définitif de la CDI sur la responsabilité des États a écarté toute référence à l'environnement et les travaux de cet organe chargé de la codification et du développement progressif du droit international n'ont jamais porté sur la mise en place d'une juridiction internationale consacrée uniquement aux atteintes graves à l'environnement.

La multiplicité des juridictions pénales internationales a indubitablement contribué à l'essor de la justice pénale internationale ${ }^{12}$, mais sans pour autant s'appesantir sur un problème d'une importance capitale pour la survie de l'humanité, en l'occurrence la répression des crimes environnementaux ou écologiques. À cet égard, il y a lieu de relever que la compétence ratione materiae des tribunaux pénaux ${ }^{13}$ ad hoc, tels que le Tribunal pénal international pour le Rwanda (TPIR) et le Tribunal pénal international pour l'ex-Yougoslavie (TPIY), y compris la CPI, n'inclut

5. Voir doc. SOC/STU/77, 22 mai 1996.

6. Voir Organisation des Nations unies, La Commission du droit international et son $\propto u v r e, 7^{\mathrm{e}}$ éd., New York, Nations unies, 2009, vol. I

7. Voir Annuaire de la Commission du droit international, 1991, vol. II, partie 1, p. 101-102.

8. Voir Annuaire de la Commission du droit international, 1995, vol. II, partie 2, p. $27, \$ 98$.

9. Ibid., p. 31, §119-120.

10. À sa $48^{\mathrm{e}}$ session, tenue du 6 mai au 26 juillet 1996. Voir CDI, « Projet d'articles sur la responsabilité des États et commentaires y relatifs adoptés par la Commission du droit international en première lecture», janvier 1997, p. 124-149 (pour le commentaire de l'article 19), en ligne: https:// legal.un.org/ilc/texts/instruments/french/commentaries/9_6_1996.pdf. Voir aussi Assemblée générale des Nations unies, 51 ${ }^{\mathrm{e}}$ session, Documents officiels, Supplément no $10, \mathrm{~A} / 51 / 10$, p. 148-174.

11. CDI, «Projet d'articles sur la responsabilité des États et commentaires y relatifs... », p. 125. À l'origine, cette disposition était l'article 18 (crimes et délits internationaux) du projet d'articles présentés par le comité de rédaction. Voir doc. A/CN.4/291 et Add.1 et 2, A/CN.4/L.243 et Add.1, p. 242.

12. Voir L'hirondelle et la tortue. Quatrièmes journées de la justice pénale internationale, J. Fernandez, O. de Frouville (dir.), Paris, Pedone, 2020; L'avenir de la justice pénale internationale, J. Albert, J.-B. Merlin (dir.), Bruxelles, Bruylant, 2018.

13. Voir J.-M. Sorel, «Les tribunaux pénaux internationaux. Ombre et lumière d'une récente grande ambition », Revue Tiers Monde, nº 205, 2011, p. 29-46. 
pas les atteintes graves à l'environnement. D'autant plus que les Statuts desdites juridictions ${ }^{14}$ ne disent rien sur la responsabilité pénale des personnes morales pour les dommages causés à l'environnement.

Pour remédier aux insuffisances à la fois de la justice pénale interne et internationale pour ce qui est de la criminalisation des atteintes à l'environnement, la doctrine et certaines organisations non gouvernementales ont axé leurs efforts sur les concepts d'écocide et d'écocrimes pour stimuler une nouvelle fois la réflexion sur la nécessité d'une juridiction pénale internationale en matière environnementale. D'autres initiatives complémentaires ont suivi dans le cadre du Conseil de l'Europe avec l'adoption de la Convention sur la protection de l'environnement par le droit pénal, ainsi que de l'Union européenne au moyen de la directive 2008/99/CE du Parlement européen et du Conseil en date du 19 novembre 2008 relative à la protection de l'environnement par le droit pénal. Et aussi au sein de l'Union africaine (UA) avec la récente Cour pénale africaine susceptible de sanctionner les atteintes à l'environnement.

L'examen de la mise en place d'une juridiction internationale spécialisée chargée de la répression des atteintes à l'environnement reste un sujet passionnant qui mérite une réflexion plus large par rapport à la CPI de La Haye. L'objectif de cet article consiste donc à réfléchir aux modalités de l'institution d'une telle juridiction et aux conséquences qui en découlent sur le plan de la responsabilité des personnes morales. Pour ce faire, nous analyserons, tout d'abord, la répression confinée des atteintes à l'environnement par la $\mathrm{CPI}(\mathrm{I})$, ensuite, la répression étendue des atteintes à l'environnement par des juridictions régionales (II), et enfin, la répression effective des atteintes à l'environnement par de nouvelles initiatives normatives et juridictionnelles (III).

\section{La répression confinée des atteintes à l'environnement par la CPI}

La CPI n'exerce aucune compétence à l'égard des crimes environnementaux. Le Statut de cette juridiction comprend un certain nombre de lacunes (A) limitant sa compétence, d'où la nécessité d'apporter certaines améliorations afin d'élargir sa compétence matérielle (B).

\section{A. Les lacunes statutaires}

La répression des atteintes à l'environnement n'est pas du ressort de la CPI. La vocation première de cette juridiction internationale permanente indépendante du système des Nations unies consiste à mettre fin à l'impunité des auteurs des crimes les plus graves qui touchent l'ensemble de la communauté internationale, notamment, suivant l'article $5 \mathrm{du}$ Statut de Rome, le génocide, les crimes contre l'humanité, les crimes de guerre et le crime d'agression.

Le Statut de Rome, adopté le 17 juillet 1998, à l'occasion de la Conférence plénipotentiaire des Nations unies sur la création de la CPI, ne fait que des références ténues à la notion d'environnement ou à la protection de la nature. Du reste, l'article 8 consacré aux crimes de guerre constitue l'unique article faisant renvoi à l'environnement naturel, dans un contexte spécifique, celui des conflits armés internationaux. Selon l'alinéa 2, b-iv de cet article, parmi les autres violations graves des lois et coutumes régissant cette catégorie de conflit, il sied de mentionner

[...] le fait de diriger intentionnellement une attaque en sachant qu'elle causera incidemment des pertes en vies humaines dans la population civile, des blessures aux personnes civiles, des dommages aux biens de caractère civil ou des dommages étendus, durables et graves à l'environnement naturel qui seraient manifestement excessifs par rapport à l'ensemble de l'avantage militaire concret et direct attendu.

La protection de l'environnement naturel n'est pas prise en compte par cette disposition réservée davantage aux populations civiles qu'aux atteintes à l'environnement. De surcroît, la même disposition ignore les conflits armés internes susceptibles de causer de sérieuses atteintes à l'environnement et impose des conditions draconiennes, en l'occurrence l'étendue, la durabilité et la gravité des dommages causés. En vérité, la protection de l'environnement en période de conflit armé est envisagée par les articles 35 et 55 du Protocole additionnel I (1977) aux quatre Conventions de Genève du 12 août 1949. Il est ainsi prohibé d'employer

[...] des méthodes ou des moyens de guerre qui sont conçus pour causer, ou dont on peut attendre qu'ils causeront, des dommages étendus, durables et graves à l'environnement naturel.

La conduite de la guerre tiendra compte de la protection de

[...] l'environnement naturel contre des dommages étendus, durables et graves. Cette protection inclut l'interdiction d'utiliser des méthodes ou moyens de guerre conçus pour causer ou dont on peut attendre qu'ils causent de tels dommages à l'environnement naturel, compromettant, de ce fait, la santé ou la survie de la population. Les attaques contre l'environnement naturel à titre de représailles sont interdites.

Remarquons que ces deux articles n'interdisent ces dommages à l'environnement que s'ils sont «étendus, durables et graves", ce qui laisse la porte ouverte à toute interprétation divergente s'agissant des atteintes à l'environnement d'un moindre degré. En définitive, il est essentiel de rappeler tout de même les limites importantes de la CPI

14. Un mécanisme international appelé à exercer les fonctions résiduelles des tribunaux pénaux ad hoc du Rwanda et de La Haye a été institué par le Conseil de sécurité de l'ONU conformément à sa résolution 1966 du 22 décembre 2010. Le mécanisme en question comprend deux divisions dont les dates d'entrée en fonction ont été fixées le $1^{\text {er }}$ juillet 2012 pour la division chargée des fonctions résiduelles du TPIR, et le $1^{\text {er }}$ juillet 2013 pour la division chargée des fonctions résiduelles du TPIY (doc. S/RES/1966 (2010), p. 2). 
sur le plan fonctionnel: la juridiction de La Haye ne peut exercer sa compétence à l'égard des crimes relevant de sa compétence que si ceux-ci ont été commis sur le territoire d'un État partie ou par un ressortissant d'un tel État. Cependant, ces deux conditions ne sont pas applicables lorsqu'une situation est déférée à la Cour par le Conseil de sécurité de l'ONU.

À cette première limite, il faut en ajouter deux autres: d'un côté, sa compétence repose entièrement sur le principe de complémentarité; en effet, la Cour ne peut intervenir que si les autorités nationales compétentes n'ont pas la volonté ou les moyens de poursuivre elles-mêmes les coupables avec la rigueur exigée ${ }^{15}$. De l'autre, elle ne peut juger que des événements qui se sont produits après l'entrée en vigueur du Statut de Rome le $1^{\text {er }}$ juillet $2002^{16}$. Par ailleurs, l'article 124 du Statut permet à un État partie de déclarer l'incompétence de la Cour, pour une période de sept ans, concernant la catégorie des crimes visés à l'article 8 , lorsqu'il est allégué qu'un crime a été commis sur son territoire ou par ses ressortissants.

\section{B. Les améliorations nécessaires}

Une juridiction pénale internationale permanente doit être en mesure de sanctionner les crimes environnementaux commis par des personnes physiques et, surtout, des personnes morales telles que les entreprises multinationales. La gravité de ces crimes est à évaluer à la lumière des conséquences néfastes engendrées sur la santé des personnes. Au demeurant, en tenant compte du critère de gravité des crimes, le Bureau du procureur de la CPI à la suite du «Document de politique générale relatif à la sélection et à la hiérarchisation des affaires ", présenté le 15 septembre 2016, a décidé d'élargir le champ d'action de la juridiction de La Haye pour s'intéresser aux crimes environnementaux. À ce sujet, le paragraphe 41 dudit document précise que:

L'impact des crimes peut s'apprécier à la lumière, entre autres, de la vulnérabilité accrue des victimes, de la terreur répandue parmi la population ou des ravages qu'ils causent sur le plan social, économique et écologique au sein des communautés concernées. Dans ce contexte, le Bureau s'intéressera particulièrement aux crimes visés au Statut de Rome impliquant ou entraînant, entre autres, des ravages écologiques, l'exploitation illicite de ressources naturelles ou l'expropriation illicite de terrains ${ }^{17}$.

À cet égard, le Bureau du procureur cherchera
[...] à la demande des États, à coopérer avec eux et à leur prêter assistance au sujet de comportements constituant des crimes graves au regard de la législation nationale, à l'instar de l'exploitation illicite de ressources naturelles, du trafic d'armes, de la traite d'êtres humains, du terrorisme, de la criminalité financière, de l'appropriation illicite de terres ou de la destruction de l'environnement ${ }^{18}$.

Bien que ce document reste muet sur la possibilité d'instaurer un «crime contre l'environnement», ce début d'intérêt par la CPI aux atteintes à l'environnement constitue un progrès ostensible, mais il reste à déterminer les modalités de la procédure à suivre afin que l'institution en question puisse prendre réellement à bras-le-corps la question des crimes environnementaux. C'est pourquoi des améliorations semblent nécessaires au Statut de Rome. Ainsi, il importe, tout d'abord, d'amender l'article $5 \mathrm{du}$ Statut pour ajouter un nouveau crime, appelé « écocide» ou «catastrophe environnementale»; ensuite, de prévoir un article pour préciser les éléments constitutifs du crime, en particulier l'élément intentionnel; enfin, de procéder à la révision ${ }^{19}$ du Statut de Rome, ce qui est une tâche ardue: le secrétaire général de l'ONU devra convoquer une conférence ouverte aux participants à l'Assemblée des États parties; l'adoption de l'amendement requiert la majorité des deux tiers (2/3) de ceux-ci, en cas d'absence de consensus.

D'autres moyens d'amélioration sont également à envisager: il en est ainsi de la création d'une chambre spécialisée ${ }^{20}$ au sein de la CPI chargée des atteintes graves à l'environnement et composée de spécialistes en la matière; de l'introduction d'un article 25 bis consacré à la responsabilité pénale des personnes morales; de l'ajout d'autres dispositions afférentes, par exemple, à la création d'un fonds spécial d'indemnisation pour les dommages graves à l'environnement, l'instauration d'une grille de peines pour les personnes morales et la mise en place d'un poste de procureur adjoint chargé de l'environnement.

Si la compétence de la CPI reste limitée en matière environnementale, d'autres juridictions peuvent sanctionner les crimes environnementaux.

\section{La répression étendue des atteintes à l'environnement par des juridictions régionales}

A contrario de la CPI, certaines juridictions régionales ont la faculté de sanctionner les atteintes à l'environnement. C'est

15. Statut de Rome, art. 17, al. 1, a et b.

16. Ibid., art. 11, al. 1 (compétence ratione temporis).

17. CPI, Bureau du procureur, «Document de politique générale relatif à la sélection et à la hiérarchisation des affaires », La Haye, 15 septembre 2016 , $\$ 41$, p. 15 .

18. Ibid., $\$ 7$, p. 5 .

19. Voir Statut de Rome, art. 121 et 123

20. À l'exemple de la Cour internationale de justice qui instaura une chambre spéciale pour les questions environnementales. Voir R. Ranjeva, «L'environnement, la Cour internationale de Justice et la Chambre spéciale pour les questions de l'environnement », Annuaire français de droit international, $\mathrm{n}^{\circ}$ 40, 1994, p. 433-441. 
le cas notamment de la Cour européenne de Strasbourg (A) et de la future Cour africaine de Malabo (B).

\section{A. La Cour européenne de Strasbourg}

Tout comme la Cour internationale de justice (CIJ) ayant contribué à travers sa jurisprudence au développement des règles et principes du droit international de l'environnement, en rappelant l'importance que revêt le respect de l'environnement pour l'ensemble des États de la communauté internationale et du genre humain, la Cour européenne des droits de l'homme a su développer pour sa part une jurisprudence riche en matière environnementale, quoique la Convention européenne de sauvegarde des droits de l'homme et des libertés fondamentales reste muette sur la notion d'environnement.

C'est par un subtil subterfuge consistant à opérer une corrélation entre les droits énoncés dans la Convention et la qualité de l'environnement que la juridiction strasbourgeoise a procédé à l'extension de son champ de compétence ratione materiae pour englober les atteintes graves à l'environnement. Dans toute une série d'affaires, la Cour de Strasbourg s'est intéressée à divers aspects touchant l'environnement.

Dans l'arrêt Tatar c. Roumanie (2009) ${ }^{21}$, une mine d'or dont le processus d'extraction utilisait du cyanure de sodium et située à proximité du domicile des requérants avait libéré dans l'écosystème environ 100 ooo $\mathrm{m}^{3} \mathrm{~d}$ 'eaux de traitement contenant des cyanures à la suite d'un accident écologique survenu en janvier 2000. La Cour a constaté que les requérants n'avaient pas établi l'existence d'un lien de causalité entre l'exposition de leur fils au cyanure de sodium et son asthme. Elle a toutefois relevé que l'activité industrielle avait continué après l'accident de janvier 2000, alors qu'aurait dû s'appliquer le principe de précaution, selon lequel l'absence de certitude compte tenu des connaissances scientifiques et techniques du moment ne saurait justifier que l'État retarde l'adoption de mesures effectives et proportionnées.

Dans l'affaire Guerra et autres c. Italie (1998) ${ }^{22}$, les requérantes habitaient à environ un kilomètre d'une usine chimique fabriquant des engrais. Un accident s'était produit ayant conduit à l'hôpital des personnes gravement intoxiquées par l'arsenic, en raison de la libération dans l'atmosphère de tonnes de substances contenant ce poison. La Cour a rappelé que des atteintes graves à l'environne- ment peuvent toucher le bien-être des personnes et les priver de la jouissance de leur domicile.

Dans l'affaire Lopez Ostra c. Espagne (1994) ${ }^{23}$, la requérante se plaignait d'une pollution émanant d'une usine de traitement de déchets de l'industrie du cuir (émanations de gaz, odeurs pestilentielles et contaminations) portant atteinte à la santé des habitants des environs. La Cour a relevé que l'État défendeur n'était pas parvenu à ménager un juste équilibre entre l'intérêt que constituait le bien-être économique de la ville - parce qu'elle accueillait une usine de traitement de déchets - et la jouissance effective par la requérante de son droit au respect de son domicile et de sa vie privée et familiale.

Dans l'affaire Giacomelli c. Italie (2006) ${ }^{24}$, la requérante se plaignait d'émissions nuisibles émanant d'une usine de traitement de "déchets spéciaux, y compris dangereux", située à trente mètres de son domicile, en alléguant que cette usine posait un risque pour sa santé et son domicile. La Cour a affirmé que, une étude d'impact sur l'environnement ayant été conduite sept ans seulement après le début de l'exploitation de l'usine, alors que la loi l'imposait avant le début de l'activité, les autorités de l'État n'avaient pas respecté la législation nationale en matière d'environnement.

Il est à remarquer, d'une part, que les arrêts de la Cour européenne sont définitifs et obligatoires pour les États parties du Conseil de l'Europe; le Comité des ministres, organe décisionnel, en assure l'application. D'autre part, l'instance strasbourgeoise contrôle aussi le respect par les États des dispositions d'un instrument révolutionnaire en matière de répression des atteintes graves à l'environnement, à savoir la Convention sur la protection de l'environnement par le droit pénal du 4 novembre $1998^{25}$.

L'originalité de cet instrument repose sur le fait qu'il érige en infractions pénales ${ }^{26}$ un certain nombre d'actes commis intentionnellement ou par négligence, tels que l'émission ou l'introduction d'une quantité de substances ou de radiations ionisantes dans l'atmosphère, le sol, les eaux; l'élimination, le traitement, le stockage, le transport, l'exportation ou l'importation illicites de déchets ; l'exploitation illicite d'une usine; la fabrication, le traitement, l'utilisation, le transport, l'exportation ou l'importation illicites de matières nucléaires, d'autres substances radioactives ou de produits chimiques dangereux. Par ailleurs, il détermine la responsabilité pénale des personnes morales ${ }^{27}$ et envisage des sanctions ${ }^{28}$ réprimant les atteintes à l'environnement, telles que l'emprisonnement et les sanctions pécuniaires et la remise en l'état de l'environnement.

21. Cour EDH, 27 janvier 2009, Tatar c. Roumanie, $\mathrm{n}^{\circ}$ 67021/01.

22. Cour EDH, 19 février 1998, Guerra et autres c. Italie, $\mathrm{n}^{\circ} 14967 / 89$.

23. Cour EDH, 9 décembre 1994, Lopez Ostra c. Espagne, n 16798/90.

24. Cour EDH, 2 novembre 2006, Giacomellic. Italie, $\mathrm{n}^{\circ}$ 59909/oo.

25. À ce jour, elle n'est pas encore en vigueur; seule l'Estonie l'a ratifiée. L'article 13, alinéa 3 exige trois instruments de ratification pour son entrée en vigueur. Voir M. G. Faure, "Vers un nouveau modèle de protection de l'environnement par le droit pénal», Revue européenne de droit de l'environnement, $\mathrm{n}^{\circ}$ 1, 2005, p. 3-19; A. Szönyi Dandachi, «La Convention sur la protection de l'environnement par le droit pénal», Revue juridique de l'environnement, $\mathrm{n}^{\circ} 3,2003$, p. 281-288.

26. Voir Convention sur la protection de l'environnement par le droit pénal, art. 2 et 4.

27. Ibid., art. 9 .

28. Ibid., art. 6 


\section{B. La Cour africaine de Malabo}

La remise en cause de la CPI par de nombreux États africains ${ }^{29}$ l'accusant de s'acharner sur le continent par l'ouverture de diverses enquêtes, surtout depuis le lancement du mandat d'arrêt à l'encontre du président soudanais Omar el-Béchir accusé de crimes graves perpétrés au Darfour, a amené ces États vers une alternative, à savoir le renforcement des pouvoirs de la Cour africaine de justice et des droits de l'homme de sorte qu'elle soit compétente pour juger et punir les crimes graves sur le plan panafricain. D'où l'adoption, le 27 juin 2014, du Protocole de Malabo $^{30}$ (Guinée équatoriale) fusionnant la Cour africaine de justice et la Cour africaine des droits de l'homme et des peuples, pour instituer la Cour africaine de justice et des droits de l'homme avec une section pénale internationale. L'objectif ouvertement affiché est la mise en place d'une justice pénale régionale ${ }^{31}$ semblable à celle de la CPI, garantissant, contrairement à cette dernière, l'immunité des chefs d'État et de gouvernement en exercice ${ }^{32}$. De ce fait, le risque d'un droit pénal régional rivalisant avec le droit international pénal ${ }^{33}$ n'est pas à exclure.

La Cour africaine pénale de Malabo exerce une compétence ratione materiae plus élargie que la CPI; elle connaît pas moins de quatorze crimes internationaux: génocide, crimes contre l'humanité, crimes de guerre, crime relatif au changement anticonstitutionnel de gouvernement, piraterie, terrorisme, mercenariat, corruption, blanchiment d'argent, traite des personnes, trafic illicite de stupéfiants, trafic illicite de déchets dangereux, exploita-

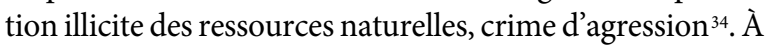
cela, il faut ajouter de manière novatrice deux compétences particulières assurées à l'égard des personnes morales impliquées souvent dans les dommages causés à l'environnement, ainsi que les crimes économiques et financiers.

La Cour pénale africaine examine les atteintes graves à l'environnement commises par les personnes morales (entreprises) sous l'angle de deux infractions: le trafic illicite de déchets dangereux, c'est-à-dire
[...] toute importation ou défaut de réimportation, tout mouvement transfrontalier ou exportation de déchets dangereux prescrit par la Convention de Bamako ${ }^{35}$ sur l'interdiction d'importer en Afrique des déchets dangereux et sur le contrôle des mouvements transfrontaliers et la gestion des déchets dangereux produits en Afrique $[\ldots]^{36}$.

Et l'exploitation illicite des ressources naturelles se manifestant par des actes graves qui affectent la stabilité d'un État, d'une région ou de l'Union africaine ${ }^{37}$; c'est le cas de l'exploitation des ressources naturelles sans respect des normes en matière de protection de l'environnement et de la sécurité des populations et du personnel; du nonrespect des normes et standards fixés par le mécanisme de certification de la ressource naturelle concernée; de la conclusion par corruption, fraude ou tromperie d'un contrat d'exploitation des ressources naturelles, etc.

En cas de responsabilité avérée des personnes morales, la Cour de Malabo rend une ordonnance contre celles qui sont coupables, en précisant les compensations auxquelles ont droit les victimes, principalement la restitution, le dédommagement et la réhabilitation ${ }^{38}$.

Les tentatives de répression des atteintes à l'environnement par des juridictions régionales démontrent bel et bien le dynamisme du droit pénal régional malgré ses limites. Qu'en est-il de la sanction des crimes environnementaux par de nouvelles actions?

\section{La répression effective des atteintes à l'environnement par de nouvelles initiatives normatives et juridictionnelles}

Les atteintes causées à l'environnement exigent une répression effective. À cet effet, de nouvelles initiatives normatives et juridictionnelles ont émergé : un projet de Convention internationale pour la lutte contre l'écocide (A) et l'instauration d'une Cour pénale internationale de l'environnement (B).

29. L'Union africaine avait appelé au retrait massif de la CPI. Cependant, le concept de retrait massif n'existe pas en droit international; les États africains ayant ratifié individuellement le Statut de Rome, ils ne peuvent en sortir qu'individuellement. Remarquons que l'Afrique constitue le bloc régional le plus nombreux à faire partie de la CPI (34 États).

30. Protocole portant amendement au Protocole portant statut de la Cour africaine de justice et des droits de l'homme, dit Protocole de Malabo. Il n'est pas encore en vigueur. Quinze ratifications sont nécessaires pour son entrée en vigueur (art. 11, al. 1).

31. Voir M. Mubiala, "Chronique de droit pénal de l’Union africaine. Vers une justice pénale régionale en Afrique», Revue internationale de droit pénal, no 3-4, vol. 83, 2012, p. 547-557.

32. Selon l'article $46 \mathrm{~A}$ bis du Protocole de Malabo, «Aucune procédure pénale n'est engagée ni poursuivie contre un chef d'État ou de gouvernement de l'UA en fonction, ou toute personne agissant ou habilitée à agir en cette qualité ou tout autre haut Responsable public en raison de ses fonctions». Cet article est en contradiction flagrante avec l'article 27, alinéa 1 du Statut de Rome disposant que: «[...] la qualité officielle de chef d'État ou de gouvernement, de membre d'un gouvernement ou d'un parlement, de représentant élu ou d'agent d'un État, n'exonère en aucun cas de la responsabilité pénale au regard du présent Statut, pas plus qu'elle ne constitue en tant que telle un motif de réduction de la peine».

33. Voir A.-M. La Rosa, Dictionnaire de droit international pénal: termes choisis, Paris, Presses universitaires de France, 1998.

34. Voir Protocole de Malabo, art. 28 A.

35. Adoptée le 30 janvier 1991. Voir F. Ouguergouz, «La Convention de Bamako sur l'interdiction d’importer en Afrique des déchets dangereux et sur le contrôle des mouvements transfrontaliers et la gestion des déchets dangereux produits en Afrique", Annuaire français de droit international, $n^{\circ} 38,1992$, p. 871-884.

36. Protocole de Malabo, art. $28 \mathrm{~L}$, al. 1.

37. Ibid., art. $28 \mathrm{~L}$ bis.

38. Ibid., art. 45 , al. 1. 


\section{A. La Convention internationale pour la lutte contre l'écocide}

En l'absence d'un instrument juridique international consacré à la lutte contre la criminalité environnementale, un groupe de juristes internationaux ${ }^{39}$ proposa des projets de conventions internationales pour la répression des écocrimes et écocides. Ceux-ci comprennent les

[...] actes illicites incriminés commis intentionnellement ou par négligence au moins grave qui créent un risque de dégradation substantielle des écosystèmes dans leur composition, leur structure ou leur fonctionnement ${ }^{40}$;

ainsi que les «actes illicites incriminés commis intentionnellement dans le cadre d'une action généralisée ou systématique et qui portent atteinte à la sûreté de la planète ${ }^{41}$. Sur ce dernier point, le crime d'écocide se rapproche du crime contre l'humanité ${ }^{42}$. En vertu de l'article 1 du projet de Convention écocide, celle-ci s'applique «aux crimes les plus graves contre l'environnement qui, en temps de paix comme en temps de conflit armé, portent atteinte à la sûreté de la planète». Les actes commis intentionnellement dans le contexte d'une action généralisée ou systématique portant atteinte à la sûreté de la planète englobent

[...] le rejet, l'émission ou l'introduction d'une quantité de substances ou de radiations ionisantes dans l'air ou l'atmosphère, les sols, les eaux ou les milieux aquatiques; la collecte, le transport, la valorisation ou l'élimination de déchets, y compris la surveillance de ces opérations ainsi que l'entretien subséquent des sites de décharge et notamment les actions menées en tant que négociant ou courtier dans toute activité liée à la gestion des déchets; l'exploitation d'une usine dans laquelle une activité dangereuse est exercée ou des substances ou préparations dangereuses sont stockées ou utilisées; la production, le traitement, la manipulation, l'utilisation, la détention, le stockage, le transport, l'importation, l'exportation ou l'élimination de matières nucléaires ou d'autres substances radioactives dangereuses; la mise à mort, la destruction, la possession ou la capture de spécimens d'espèces de faune et de flore sauvages protégées ou non ${ }^{43}$.

Ils doivent impérativement causer

[...] une dégradation étendue, durable et grave de l'air ou de l'atmosphère, des sols, des eaux, des milieux aquatiques, de la faune ou de la flore, ou de leurs fonctions écologiques; ou la mort, des infirmités permanentes ou des maladies incurables graves à une population ou [...] déposs[éder] durablement cette dernière de ses terres, territoires ou ressources ${ }^{44}$.

Le projet de Convention écocide prévoit, à juste titre, l'imprescriptibilité du crime d'écocide ${ }^{45}$ et la responsabilité pénale des personnes morales ${ }^{46}$, c'est-à-dire

[...] toute entité ayant la personnalité juridique en vertu du droit applicable, exception faite des États ou des entités publiques dans l'exercice de leurs prérogatives de puissance publique et des organisations internationales publiques ${ }^{47}$.

À cet effet, tout État partie adopte des mesures législatives internes afin qu'une personne morale puisse être tenue pénalement responsable du crime d'écocide, lorsque celui-ci a été commis, pour son compte, par toute personne exerçant un pouvoir de direction en son sein, agissant soit individuellement, soit en tant que membre d'un organe de la personne morale ${ }^{48}$.

Les sanctions à l'encontre des personnes morales sont envisagées par l'article 7 , alinéas 1 et 2 du projet de Convention écocide. Elles portent de manière détaillée sur les amendes; la dissolution; la fermeture temporaire ou définitive des locaux ou établissements de la personne morale; le retrait de licences, autorisations ou concessions; l'interdiction de recevoir des subventions et des financements publics et de contracter avec les administrations publiques; la publication de la condamnation; la nomination d'un mandataire de justice.

L'ensemble de ces sanctions est déterminé en tenant compte prioritairement de la réparation du dommage et de l'indemnisation des victimes, selon un certain nombre de critères ${ }^{49}$, tels que le profit économique tiré de l'infraction, y compris dans ce cas les économies ayant pu résulter de la non-adoption de mesures de protection de l'environnement; l'absence ou l'insuffisance des mesures de contrôle internes qui auraient permis la prévention de l'infraction; la réitération d'infractions contre l'environnement au sein de ou par la personne morale et la collaboration de la personne morale. La réparation des dommages causés par les actes illicites des personnes morales prend la forme de mesures de remise en état; de dommages et intérêts; de programmes de conformité; de provisionnement du Fonds pour l'environnement; de mesures de développement local; d'excuses aux communautés lésées en tant que mesures de réparation

39. Des écocrimes à l'écocide: le droit pénal au secours de l'environnement, L. Neyret (dir.), préface de M. Delmas-Marty, Bruxelles, Bruylant, 2015. La recherche a aussi abouti à la formulation de trente-cinq propositions pour mieux sanctionner les crimes contre l'environnement; voir la partie III, p. 445-451.

40. Ibid., p. 452 ("Tableau comparatif des infractions environnementales»)

41. Ibid. Voir aussi le projet de Convention écocide, art. 2, al. 1, disponible en ligne: http://blog.uclm.es/repmult/files/2019/12/o2_PROJET-DECONVENTION-CONTRE-L\%E2\%80\%99\%C3\%89COCIDE.pdf.

42. Voir M. Delmas-Marty, L. Neyret, I. Fouchard, E. Fronza, Le crime contre l'humanité, Paris, Presses universitaires de France, 2018 , p. 128.

43. Projet de Convention écocide, art. 2, al. 1, a, b, c, d, e.

44. Ibid., art. 2, al. 2, a et b.

45. Ibid., art. 4

46. La responsabilité pénale des personnes physiques est prévue par l'article 3 du projet Convention écocide.

47. Projet de Convention écocide, art. 5 , al. 4

48. Ibid., art. 5, al. 1

49. Ibid., art. 8 
symboliques adaptées à la dimension culturelle du dommage environnemental ${ }^{50}$.

Pour ce qui est des personnes physiques, les sanctions ${ }^{51}$ doivent être efficaces, proportionnées et dissuasives, tout en garantissant la réparation des dommages à l'environnement et l'indemnisation des victimes. Les peines encourues peuvent prendre la forme de peines d'emprisonnement, d'amendes et de confiscation des profits, biens et avoirs tirés directement ou indirectement du crime, sans préjudice des droits des tiers de bonne foi. Le choix et la détermination de la gravité de la sanction reposent sur plusieurs critères, en l'occurrence le profit économique tiré de l'infraction, y compris dans ce cas les économies ayant pu résulter de la non-adoption de mesures de protection de l'environnement; la position hiérarchique de l'auteur de l'infraction; la prompte réparation du dommage et l'indemnisation des victimes; le caractère organisé du crime. Les mesures de réparation des dommages causés par les personnes physiques sont semblables à celles des personnes morales.

Les autres dispositions innovantes ${ }^{52}$ du projet de Convention écocide touchent la création du poste de procureur international de l'environnement ${ }^{53}$, d'une Cour pénale internationale de l'environnement ${ }^{54}$ et d'un Groupe de recherche et d'enquête pour l'environnement (GREEN) ${ }^{55}$ chargé d'effectuer des constatations de faits matériels susceptibles de correspondre à la définition du crime d'écocide et de formuler des avis sur la criminalité environnementale internationale.

Certes, le projet de Convention internationale pour la lutte contre l'écocide est ambitieux ${ }^{56}$ de par l'enjeu recherché visant à réprimer les atteintes graves à l'environnement, néanmoins sa concrétisation suscite certaines interrogations. En effet, le mécanisme d'application envisagé est purement politique, l'Assemblée des États parties, avec un mode de prise de décisions souvent difficile à atteindre, le consensus; alors qu'il aurait été plus idoine de confier la tâche de contrôle à un organe ou un groupe d'experts indépendants. Plus généralement, le projet de Convention ne dit rien au sujet du nombre d'instruments de ratification requis pour son entrée en vigueur, ni sur la question de la possibilité de formuler des réserves par les États parties. Enfin, le processus d'adoption du texte écocide à l'ONU exige aussi la convocation par le secrétaire général d'une conférence plénipotentiaire et la désignation d'un groupe de travail pour assurer la rédaction de ses articles.

\section{B. La Cour pénale internationale de l'environnement}

L'initiative d'une juridiction pénale internationale dédiée à l'environnement revient à un groupement d'organisations ${ }^{57}$ qui lança au sein du Parlement européen, le 30 janvier 2014, la Charte de Bruxelles pour la création d'un Tribunal pénal européen et d'une Cour pénale internationale de l'environnement et de la santé.

Les promoteurs de la Charte ont constaté que l'utilisation excessive des ressources contribue à la destruction d'écosystèmes dont le fonctionnement assure le maintien et le développement de la vie; et que l'usage de produits toxiques et en particulier les perturbateurs endocriniens et les produits cancérigènes, mutagènes et reprotoxiques affecte sérieusement la santé humaine. De ce fait, il importe d'agir de manière urgente sur le plan juridique afin de garantir la préservation de l'environnement avec des sanctions effectives, proportionnées et dissuasives. C'est pourquoi, l'accès effectif à la justice s'avère indispensable pour réprimer les dommages portés aux ressources, à la nature et aux humains.

Reconnaissant «le droit inaliénable de l'homme à un environnement sain et le risque d'irréversibilité du niveau atteint par la perte de biodiversité ${ }^{58}$, la Charte conçoit un processus en trois étapes ${ }^{59}$ pour l'instauration de juridictions consacrées à l'environnement. De prime abord, un Tribunal moral pour juger les responsables des crimes et délits environnementaux mettant en péril les ressources planétaires et la santé humaine par la société civile. Ensuite, la création d'un Tribunal pénal européen de l'environnement et de la santé par la modification des statuts de la Cour de justice de l'Union européenne; la reconnaissance de la nécessaire sanction pénale des délits environnementaux (directive 2008/99/CE) et la mise en place d'un Parquet pénal européen. Enfin, l'institution d'une Cour pénale internationale de l'environnement

50. Projet de Convention écocide, art. 7, al. 3 .

51. Ibid., art. 6 , al. 1,2 et 3 .

52. Elles concernent les enquêtes et poursuites, l'extradition, l'entraide judiciaire et la coopération internationale. Voir projet de Convention écocide, art. $11,14,15$ et 16

53. Projet de Convention écocide, art. 17.

54. Ibid., art. 18 .

55. Ibid., art. 20.

56. La rédaction de certains articles s'inspire tout de même des dispositions de la directive 2008/99/CE du Parlement européen et du Conseil du 19 novembre 2008 relative à la protection de l'environnement par le droit pénal. Voir, par exemple, les articles 3 (infractions), 5 (sanctions) et 6 (responsabilité des personnes morales) de la directive. Voir aussi L. Krämer, "Le droit répressif et le droit de l'environnement européen ", Revue juridique de l'environnement, numéro spécial, 2014, p. 75-93; D. Roets, « Naissance du droit pénal européen de l'environnement (à propos de la directive 2008/99/CE du Parlement européen et du conseil du 19 novembre 2008 relative à la protection de l'environnement par le droit pénal)», Revue européenne de droit de l'environnement, $\mathrm{n}^{\circ}$ 3, 2009, p. 271-283.

57. End Ecocide in Europe, Tribunal international de conscience des crimes relatifs à la nature, Académie internationale des sciences environnementales, Fondation Lelio Basso, etc.

58. Préambule de la Charte de Bruxelles.

59. Ibid., principes 1,2 et 3 . 
et de la santé avec la reconnaissance de «la catastrophe environnementale comme l'une des incriminations des crimes contre l'humanité permettant de poursuivre les responsables ayant agi de façon intentionnelle». Ainsi, ce crime de catastrophe environnementale

[...] permettrait d'obtenir une protection effective internationale des écosystèmes, dans l'esprit des précédents jurisprudentiels de nature civile émanant de la Cour internationale de Justice (Trail Case, conflit du canal de Corfou) dont résulte une règle de droit international coutumier selon laquelle: «aucun État n'a le droit d'utiliser ou permettre qu'on utilise son propre territoire de telle manière à provoquer des dommages", réaffirmée par le principe $n^{\circ} 21$ de la Déclaration de Stockholm de 1972 et dans le principe $\mathrm{n}^{\circ} 2$ de la Conférence de Rio de Janeiro de $1992^{60}$.

L'idée d'une Cour pénale internationale de l'environnement complémentaire des juridictions nationales avec un procureur international de l'environnement indépendant fut avancée par les initiateurs du projet de Convention internationale pour la lutte contre l'écocide. L'Assemblée des États parties à la Convention choisira pour un mandat de cinq ans le procureur international compétent pour

[...] enquêter et rassembler des preuves relatives à des actes présumés d'écocide portés à la connaissance de son Bureau, par les autorités nationales des États parties, par les institutions régionales et internationales intéressées par la lutte contre la criminalité environnementale, par la société civile... ${ }^{61}$.

Elle désignera aussi des procureurs correspondants ${ }^{62}$ nationaux pour l'assister dans ses tâches.

La démarche concrète pour la mise en place d'une juridiction pénale internationale dévouée à la répression des atteintes à l'environnement passe par la convocation d'une conférence internationale sous l'égide des Nations unies. Le secrétaire général de l'ONU désignera alors un groupe de travail composé d'États parties pour recueillir l'avis des autres membres et rédiger les dispositions du Statut de la future juridiction. Dans cette intention, la question de la responsabilité internationale pour dommages causés à l'environnement doit être davantage mise en lumière, tout en rappelant l'importance des principes 21 et 22 de la Conférence de Stockholm des Nations unies sur l'environnement de juin $1972^{63}$. Ceux-ci méritent d'être mentionnés:

- Conformément à la Charte des Nations unies et aux principes du droit international, les États ont le droit souverain d'exploiter leurs propres ressources selon leur politique d'environnement et ils ont le devoir de faire en sorte que les activités exercées dans les limites de leur juridiction ou sous leur contrôle ne causent pas de dommage à l'environnement dans d'autres États ou dans des régions ne relevant d'aucune juridiction nationale.

- Les États doivent coopérer pour développer encore le droit international en ce qui concerne la responsabilité et l'indemnisation des victimes de la pollution et d'autres dommages écologiques que les activités menées dans les limites de la juridiction de ces États ou sous leur contrôle causent à des régions situées au-delà des limites de leur juridiction ${ }^{64}$.

La jurisprudence de la CIJ s'est fait l'écho de ces principes: dans l'affaire des usines de pâte à papier sur le fleuve Uruguay (Argentine c. Uruguay) ${ }^{65}$, l'organe judiciaire de l'ONU a affirmé que

[...] le principe de prévention, en tant que règle coutumière, trouve son origine dans la diligence requise («due diligence») de l'État sur son territoire. Il s'agit de «l'obligation, pour tout État, de ne pas laisser utiliser son territoire aux fins d'actes contraires aux droits d'autres États» [...]. En effet, l'État est tenu de mettre en œuvre tous les moyens à sa disposition pour éviter que les activités qui se déroulent sur son territoire, ou sur tout espace relevant de sa juridiction, ne causent un préjudice sensible à l'environnement d'un autre État ${ }^{66}$.

Cette obligation relève désormais du corps de règles du droit international de l'environnement.

Dans un autre litige relatif au projet GabčíkovoNagymaros (Hongrie c. Slovaquie) ${ }^{67}$, la CIJ a rappelé que, dans le domaine de la protection de l'environnement,

[...] la vigilance et la prévention s'imposent en raison du caractère souvent irréversible des dommages causés à l'environnement et des limites inhérentes au mécanisme même de réparation de ce type de dommages ${ }^{68}$.

Et d'ajouter que:

La réparation doit «autant que possible» effacer toutes les conséquences de l'acte illicite. En l'espèce, les conséquences des actes illicites commis par les deux parties seront effacées «autant que possible» si ces dernières reprennent leur coopération pour l'utilisation des ressources en eau partagées du Danube et si le programme pluridimensionnel d'utilisation, de mise en valeur et de protection du cours d'eau, en tant qu'unité unique coordonnée, est réalisé de manière équitable et raisonnable. Ce que peuvent faire les

60. Ibid., principe 3

61. Projet de Convention écocide, art. 17, al. 2.

62. Ibid., art. 17, al. 3 .

63. Voir A. C. Kiss, J.-D. Sicault, «La Conférence des Nations unies sur l'environnement (Stockholm, 5-16 juin 1972)», Annuaire français de droit international, $\mathrm{n}^{\circ} 18,1972$, p. 603-628.

64. Rapport de la Conférence des Nations unies sur l'environnement, Stockholm, 5-16 juin 1972, principes 21 et 22.

65. CIJ, arrêt du 20 avril 2010, Usines de pâte à papier sur le fleuve Uruguay (Argentine c. Uruguay), Recueil, 2010, p. 14 sq. Voir L. Vatna, «L'affaire des Usines de pâte à papier sur le fleuve Uruguay (Argentine c. Uruguay) : un nouveau différend environnemental devant la Cour internationale de justice», Revue québécoise de droit international, $\mathrm{n}^{\circ}$ 22-2, 2009, p. 25-55.

66. CIJ, arrêt du 20 avril 2010, Usines de pâte à papier..., p. 55-56, \$101.

67. CIJ, arrêt du 25 septembre 1997, Projet Gabčíkovo-Nagymaros (Hongrie c. Slovaquie), Recueil, 1997, p. 7 sq.

68. Ibid., p. $78, \$ 140$, al. 3 . 
parties, c'est rétablir une gestion conjointe de ce qui reste du projet. À cette fin, il leur est loisible, d'un commun accord, de conserver les ouvrages de Čunovo, en apportant des changements dans le mode d'exploitation du système pour ce qui est de la répartition de l'eau et de l'électricité, et de ne pas construire les ouvrages de Nagymaros ${ }^{69}$.

Les formes de la réparation intégrale du préjudice causé par le fait illicite ont été précisées par la CDI, dans son «Projet d'articles sur la responsabilité de l'État pour fait internationalement illicite $»^{70}$. Il s'agit dans l'ordre ${ }^{71} \mathrm{de}$ manière séparée ou conjointe de la restitution consistant dans le rétablissement de la situation qui existait avant que le fait illicite ne soit commis; de l'indemnisation couvrant tout dommage susceptible d'évaluation financière, y compris le manque à gagner dans la mesure où celui-ci est établi; de la satisfaction se manifestant par la reconnaissance de la violation, une expression de regrets, des excuses formelles ou tout autre moyen approprié.

La prise en compte des enjeux environnementaux par la communauté internationale ne cesse de croître, car l'environnement n'est plus «une abstraction, mais bien l'espace où vivent les êtres humains et dont dépendent la qualité de leur vie et leur santé, y compris pour les générations à venir ${ }^{72}$. La répression des atteintes graves à l'environnement devient donc une action légitime et inéluctable à partir du moment où les crimes environnementaux peuvent sérieusement menacer la paix et le développement durable et font partie désormais des formes les plus lucratives d'activités criminelles transnationales ${ }^{73}$.

La construction d'un véritable ordre public environnemental ou écologique ${ }^{74}$ passe concrètement par l'institution d'une juridiction internationale spécialisée chargée de la répression des atteintes graves à l'environnement. Les diverses initiatives préconisées à ce jour (extension de la compétence de la CPI, projet de Convention écocide, Cour pénale internationale de l'environnement...) pour une justice pénale internationale effective en la matière sont louables et vont dans le bon sens, mais le chemin à parcourir est encore long et semé d'embûches: tout dépend par excellence de la bonne volonté des États et de leur désir de lancer le débat dans un cadre multilatéral sous l'égide des Nations unies pour édifier un droit international commun de l'environnement en vue de préserver la planète et les générations futures.

69. CIJ, arrêt du 25 septembre 1997, Projet Gabčíkovo-Nagymaros..., p. 80, \$150.

70. Voir Assemblée générale des Nations unies, $56^{\mathrm{e}}$ session, Documents officiels, Supplément no $10, \mathrm{~A} / 56 / 10$; Assemblée générale des Nations unies, $56^{\mathrm{e}}$ session, résolution 56/83, 12 décembre 2001. Voir aussi A. Pellet, «Les articles de la CDI sur la responsabilité de l'État pour fait internationalement illicite. Suite - et fin?», Annuaire français de droit international, n 48, 2002, p. 1-23.

71. Voir «Projet d'articles...», art. 34 à 37.

72. Voir CIJ, avis consultatifs, 8 juillet 1996, Licéité de la menace ou de l'emploi d’armes nucléaires, Recueil, 1996, p. 241-242, \$29.

73. Selon un rapport conjoint PNUE-INTERPOL (Programme des Nations unies pour l'environnement et Organisation internationale de police criminelle, «La valeur des atteintes à l'environnement a augmenté de $26 \%$ », 4 juin 2016), la valeur monétaire tirée de ces crimes se situait en 2016 entre 91 et 258 milliards de dollars. Voir PNUE, The Rise of Environmental Crime: A Growing Threat to Natural Resources Peace, Development and Security, 2016, p. 7, en ligne: http://wedocs.unep.org/handle/20.500.11822/7662.

74. Voir N. Belaïdi, La lutte contre les atteintes globales à l'environnement: vers un ordre public écologique?, Bruxelles, Bruylant, 2008. 